\title{
Multimodality cardiac computed tomography angiography and magnetic resonance with clinical-grade scanners provide robust assessment of cardiac morphology and function in rabbits
}

\author{
Gitsios Gitsioudis $^{1,2,3}$, Maximilian Nunninger ${ }^{1,2}$, Anna Missiou ${ }^{1,2,4}$, Peter Wolf ${ }^{1,2}$, Hugo A. Katus ${ }^{1,2}$, \\ Grigorios Korosoglou ${ }^{1,2}$
}

${ }^{1}$ Department of Cardiology, University of Heidelberg, Heidelberg, Germany; ${ }^{2}$ Department of Cardiology, University of Heidelberg, Heidelberg, Germany DZHK (German Centre for Cardiovascular Research), Partner Site Heidelberg, Heidelberg, Germany; ${ }^{3}$ Department of Internal Medicine, Cardiology, Hospital of Bietigheim-Vaihingen, Academic Hospital of the University of Heidelberg, Bietigheim, Germany; ${ }^{4}$ Department of Cardiology, Clinic of Ludwigsburg, Academic Hospital of the University of Heidelberg, Ludwigsburg, Germany

Contributions: (I) Conception and design: G Gitsioudis, M Nunninger, G Korosoglou; (II) Administrative support: G Gitsioudis, HA Katus, G Korosoglou; (III) Provision of study materials or patients: G Gitsioudis, G Korosoglou; (IV) Collection and assembly of data: G Gitsioudis, M Nunninger, P Wolf, A Missiou, G Korosoglou; (V) Data analysis and interpretation: G Gitsioudis, M Nunninger, A Missiou, G Korosoglou; (VI) Manuscript writing: All authors; (VII) Final approval of manuscript: All authors.

Correspondence to: Gitsios Gitsioudis, Dr.med, Dipl.Phys. Department of Internal Medicine, Cardiology, Hospital of Bietigheim-Vaihingen, Academic Hospital of the University of Heidelberg, Riedstraße 12, 74321 Bietigheim, Germany. Email: gitsioudis_correspondence@web.de.

Background: Non-invasive computer tomography (CT)- and magnetic resonance (MR)-based cardiac imaging still remains challenging in rodents. To investigate the robustness of non-invasive multimodality cardiac imaging in rabbits using clinical-grade CT and MR scanners.

Methods: A total of 16 rabbits $(2.7-4.0 \mathrm{~kg})$ serially underwent cardiac-gated imaging using a clinical-grade 256-row CT and a 1.5 Tesla MR-scanner at baseline and at 4-month follow-up (16 1 weeks). Image analysis included image quality (5-grade scale), left ventricular (LV) volumes, LV stroke volume, LV diameters, LV wall thickness and ejection fraction (LVEF).

Results: Cardiac MR (CMR) and CT angiography (CTA) provide images with an overall good image quality (excellent or good quality: CMR $82 \%$ vs. CTA 78\%, P=0.68). Linear regression analysis demonstrated a good correlation of all diameters (diam.) and volumes (vol.) as assessed by CTA and CMR (diam.: r=0.9, 95\% CI: 0.8-0.9; vol.: r=0.8, 95\% CI: 0.6-0.9; P<0.0001 for both). CTA-based volumetric analysis revealed slightly higher LVEF values as compared to CMR (CTA: $64 \% \pm 1 \%$, CMR: $59 \% \pm 1 \%, \mathrm{P}=0.002$ ). Analysis of inter-/intra-observer agreement demonstrated excellent agreements for diameters (CMR: 98.5\%/98.7\%; CTA: 98.2\%/97.4\%) and volumes (CMR: 99.9\%/98.8\%; CTA 98.7\%/98.7\%). Finally, serial CMR- and CTA-based assessment of cardiac diameters and volumes delivered excellent intersession agreements of baseline versus follow-up data (diam.: CMR: r=0.89; CTA: r=0.92; vol.: CMR: r=0.87; CTA: r=0.96, $\mathrm{P}<0.0001$ for all).

Conclusions: Multimodality non-invasive assessment of cardiac function and aortic hemodynamics is feasible and robust in rabbits using clinical-grade and MR and CT scanners. These imaging modalities could improve serial cardiac assessment for disease monitoring in preclinical settings.

Keywords: Cardiac magnetic resonance (CMR); cardiac-gated computer tomography angiography (cardiac-gated CTA); velocity-encoded phase contrast magnetic resonance imaging (velocity-encoded phase contrast MRI); rabbits

Submitted May 20, 2019. Accepted for publication Aug 26, 2019.

doi: $10.21037 /$ jtd.2019.10.46

View this article at: http://dx.doi.org/10.21037/jtd.2019.10.46 


\section{Introduction}

Rodents and rabbits are the most widespread preclinical models to study cardiovascular disease and therapeutic interventions. However, non-invasive assessment of cardiac function and hemodynamics in these animals still remains challenging due to very high heart rates (150-300/sec) and small heart sizes $(3-10 \mathrm{~g})(1)$.

In general, 2-dimensional imaging techniques have geometrical limitations to assess cardiac morphology and function. Although, echocardiography is the working horse for routine non-invasive cardiac diagnostics (2-6) the assessment of global functional parameters and Dopplerbased hemodynamics are usually approximated from highly operator dependent $2 \mathrm{D}$ approaches $(7,8) .3$ or $4 \mathrm{D}$ echo techniques are rarely available and require expensive instruments (>300,000\$) with limited availability (9).

Cardiac-gated computed tomography angiography (CTA) on the other hand, enables for highly reproducible time resolved 3D cardiac views with excellent temporal and spatial resolution and accurate delineation of the contrastenhanced blood pool (10-12). However, CTA intrinsically provides very limited information on hemodynamics. Cardiac magnetic resonance (CMR) enables for both time resolved cardiovascular imaging with excellent intrinsic soft tissue contrast, and assessment of cardiac and vascular hemodynamics $(13,14)$. To apply clinical-grade MR or CT scanners in rabbits, mostly customized radiofrequency coils or ultra-high magnetic field strengths ( $\geq 4.7$ Tesla) are needed, which is accompanied with significant costs (15-20).

Therefore, this serial imaging study aimed to investigate the ability and to compare clinical-grade CT and MR scanners in the setting of an experimental rabbit model using suited acquisition protocols to assess cardiac morphology, function and aortic hemodynamics.

\section{Methods}

\section{Animals}

A total of 16 rabbits $(2.7-4.0 \mathrm{~kg}, 13.0 \pm 0.9$ months at baseline) underwent both CMR imaging, immediately followed by CTA using clinical-grade MR and CT scanners at baseline and follow-up (16 \pm 1 weeks, Figure $1 A)$.

\section{MR scanner}

All experiments were conducted with a clinical-grade stateof-the-art 1.5 Tesla MR scanner (Philips Achieva, Best,
The Netherlands) using a 32-element cardiac phased-array receiver coil.

\section{MR imaging protocol}

All animals underwent a standardized serial CMR imaging protocol, which is illustrated in Figure 1B. First, we acquired a survey, followed by a 3D T1-weighted acquisition for detailed planning of the subsequent CINE-bSSFP 4-chamber, short axis (SA) views and $2 \mathrm{D}$ velocity-encoded phase-contrast MR acquisitions. Details on imaging parameters for CINE-bSSFP and 2D CINE velocityencoded PCI-MRI are provided in the Supplementary file.

\section{CMR image analysis}

All CMR image analysis was performed using commercially available software (View Forum, Version 5.1, Philips Medical Systems, Best, The Netherlands). LV deformation analysis (strain and strain rate) were conducted using dedicated software (TomTec Imaging Systems, Munich, Germany). Image quality of CMR data sets were analyzed in random order. Details are provided in the Supplementary file.

\section{CT imaging protocol and radiation dosage}

Details for animal preparation and monitoring are presented in the Supplementary file. All experiments were serially conducted using a clinical-grade CT-scanner with a $2 \mathrm{~mm} \times 128 \mathrm{~mm} \times 0.625 \mathrm{~mm}$ detector collimation and dynamic z-focal spot (iCT, Philips Medical Systems, Best, The Netherlands). To minimize cardiac motion artifacts, we applied retrospective vector-ECG gating. The detailed CT imaging protocol is illustrated in Figure 1C. First, we acquired a topogram, followed by a native CT imaging. For CTA, contrast material was applied using a triphasic application protocol to avoid beam-hardening artefacts (21): first, a bolus of $3 \mathrm{~mL}$ of contrast agent (Imeron ${ }^{\circledR} 400$, Bracco Imaging, Germany) was injected, followed by a mix of contrast material $(3 \mathrm{~mL})$ and saline $(8 \mathrm{~mL})$ and a final application of a bolus of $3 \mathrm{~mL}$ saline flush at a flow of $1.5 \mathrm{~mL} / \mathrm{s}$. The scan started automatically using a bolus tracking with a region of interest placed in the descending aorta (threshold level $110 \mathrm{HU}$ ). The entire volume was acquired with simultaneous ECG recording (Figure $2 A, B$ ).

The following imaging parameters were used for CTA imaging: tube voltage $120 \mathrm{kV}$; effective tube current-time 
A

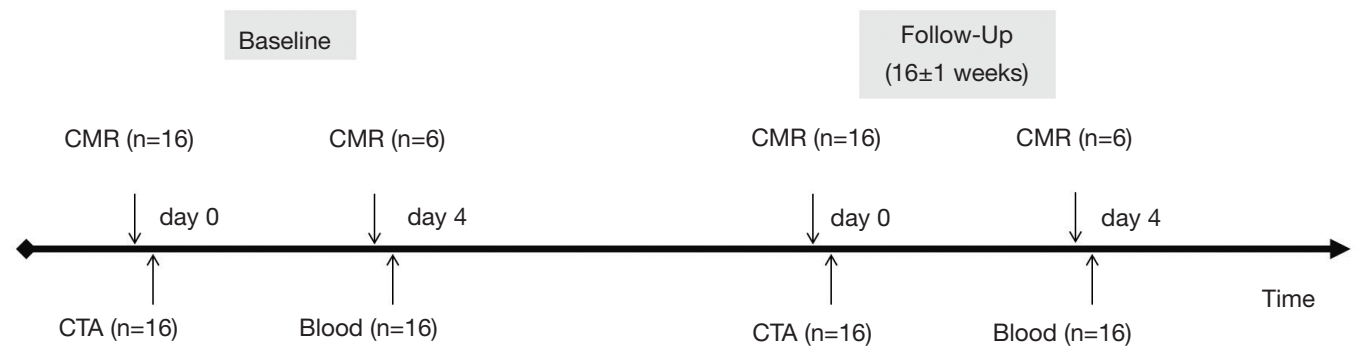

B
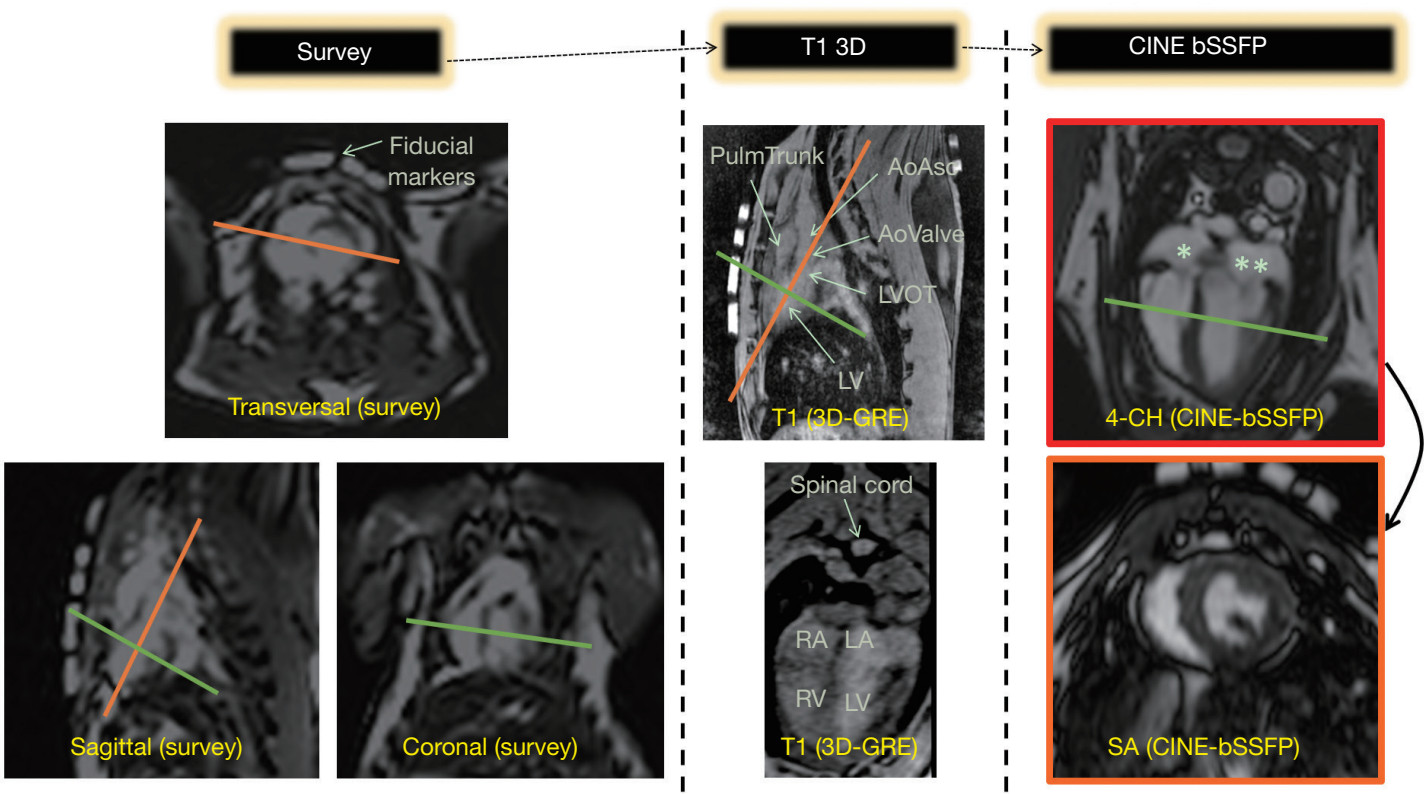

C

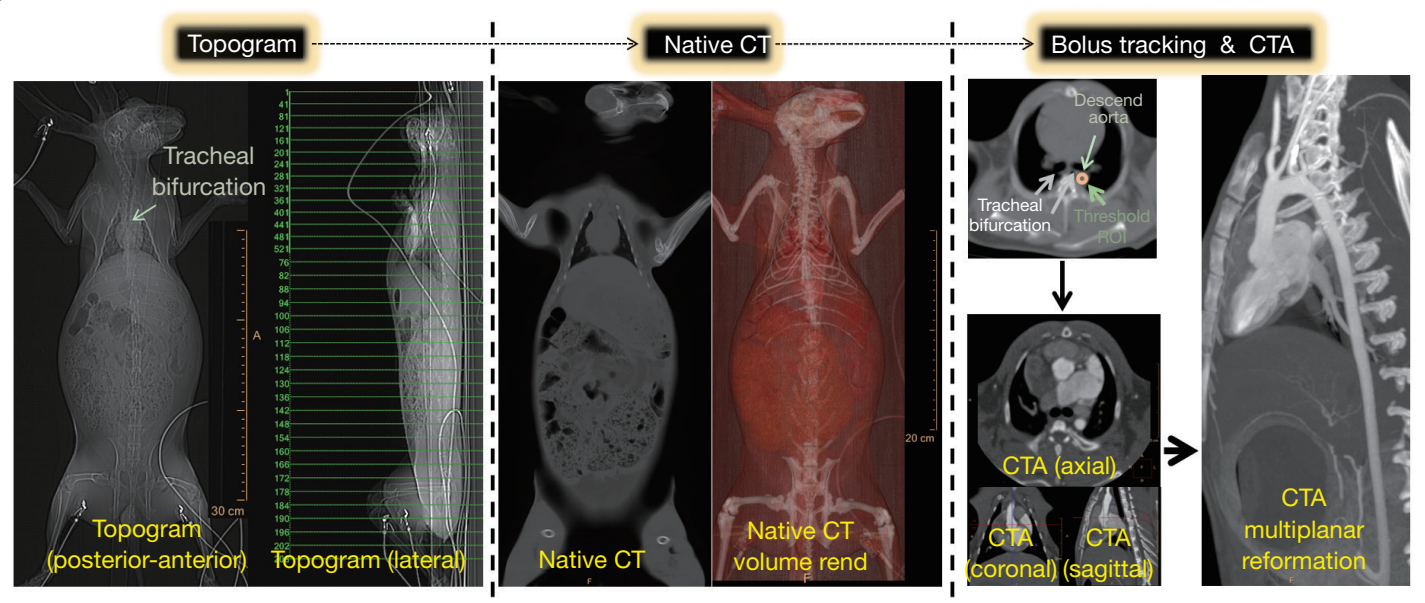

Figure 1 Overview of study design and cardiac imaging modalities. (A) Flow diagram of the serial cardiac imaging study design; (B) imaging protocol for CMR imaging and detailed planning of 4-chamber (4-Ch) view and SA view; (C) imaging protocol for CTA. CMR, cardiac magnetic resonance; CTA, cardiac-gated computed tomography angiography; SA, short-axis. 
A
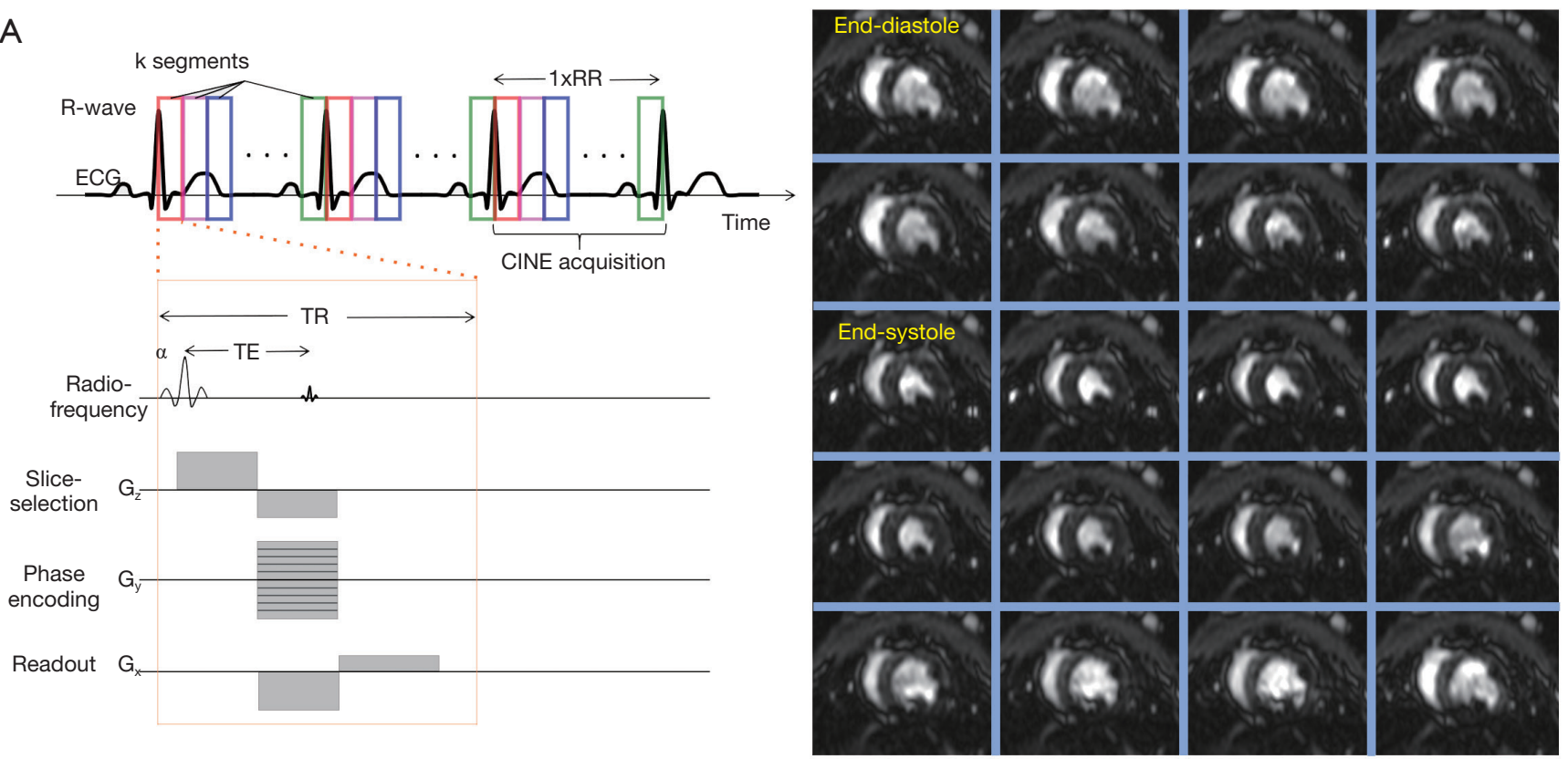

B
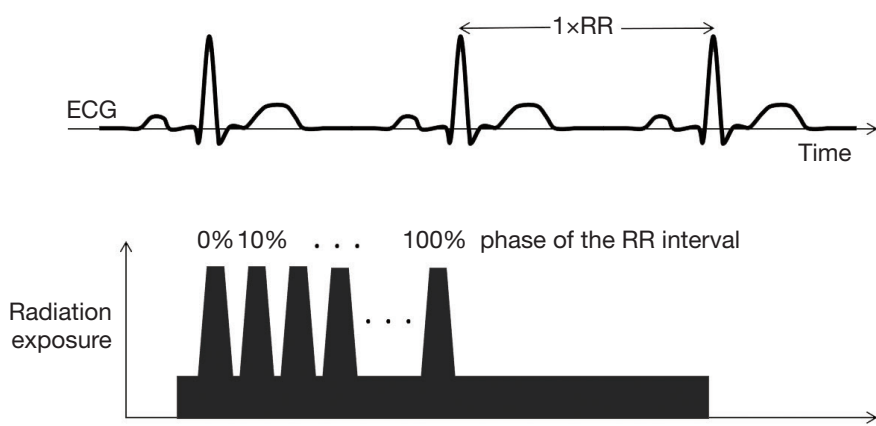

Helical scan with dose modulation

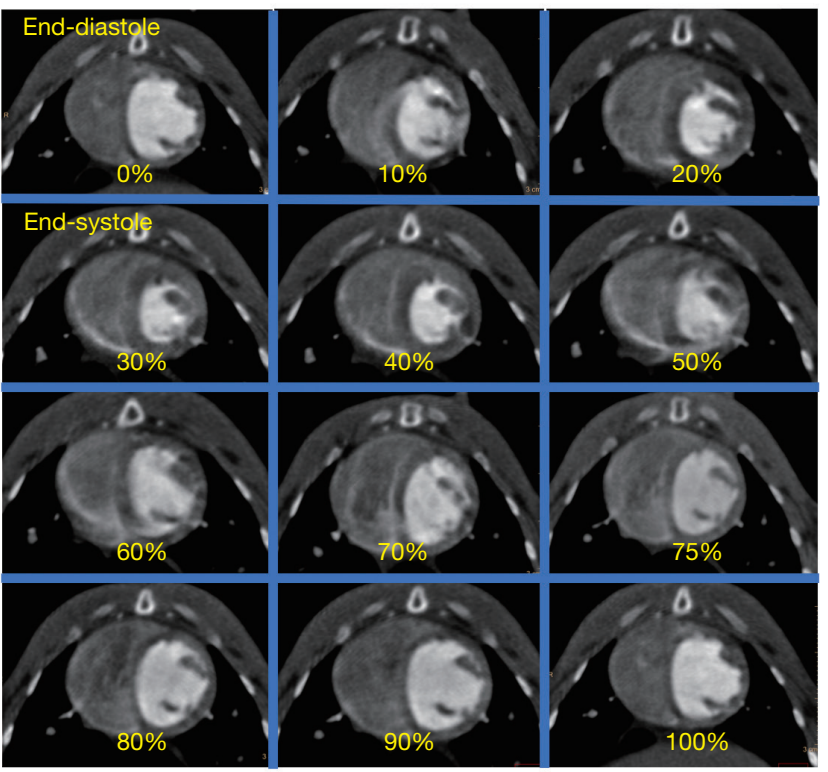

Figure 2 Imaging protocols of cardiac magnetic resonance (CMR) and CTA imaging. (A) Pulse sequence diagram for phase-modulated proton excitation CINE-bSSFR acquisition (left). The corresponding CMR images at different time-points within one RR-cycle are presented on the right; (B) schematic diagram for the retrospective CTA (left). The corresponding CTA images at different time-points within one RR-cycle are presented on the right. CTA, cardiac-gated computed tomography angiography.

product of $400 \mathrm{mAs}$ per slice; gantry rotation time $0.27 \mathrm{~s}$; pitch 0.2 ; image matrix $1,024 \times 1,024$. Reconstructions were performed routinely for all phases $(0-100 \%)$ with a $10 \%$ increment. The total radiation dose (CTDIvol) was obtained from the examination protocol of the system.

\section{CT image analysis}

All CT image analysis was performed on a dedicated workstation (Extended Brilliance Workspace 4.0, Philips Medical Systems) and on commercially available software (Osirix MD, Version 2.8., Pixmeo Sarl, Bernex, Switzerland). Image quality of $\mathrm{CT}$ data sets were analyzed in random 
Table 1 Serial assessment of cardiac diameters and volumes in cardiac CINE-bSSFP CMR and CTA in all animals ( $\mathrm{n}=16)$

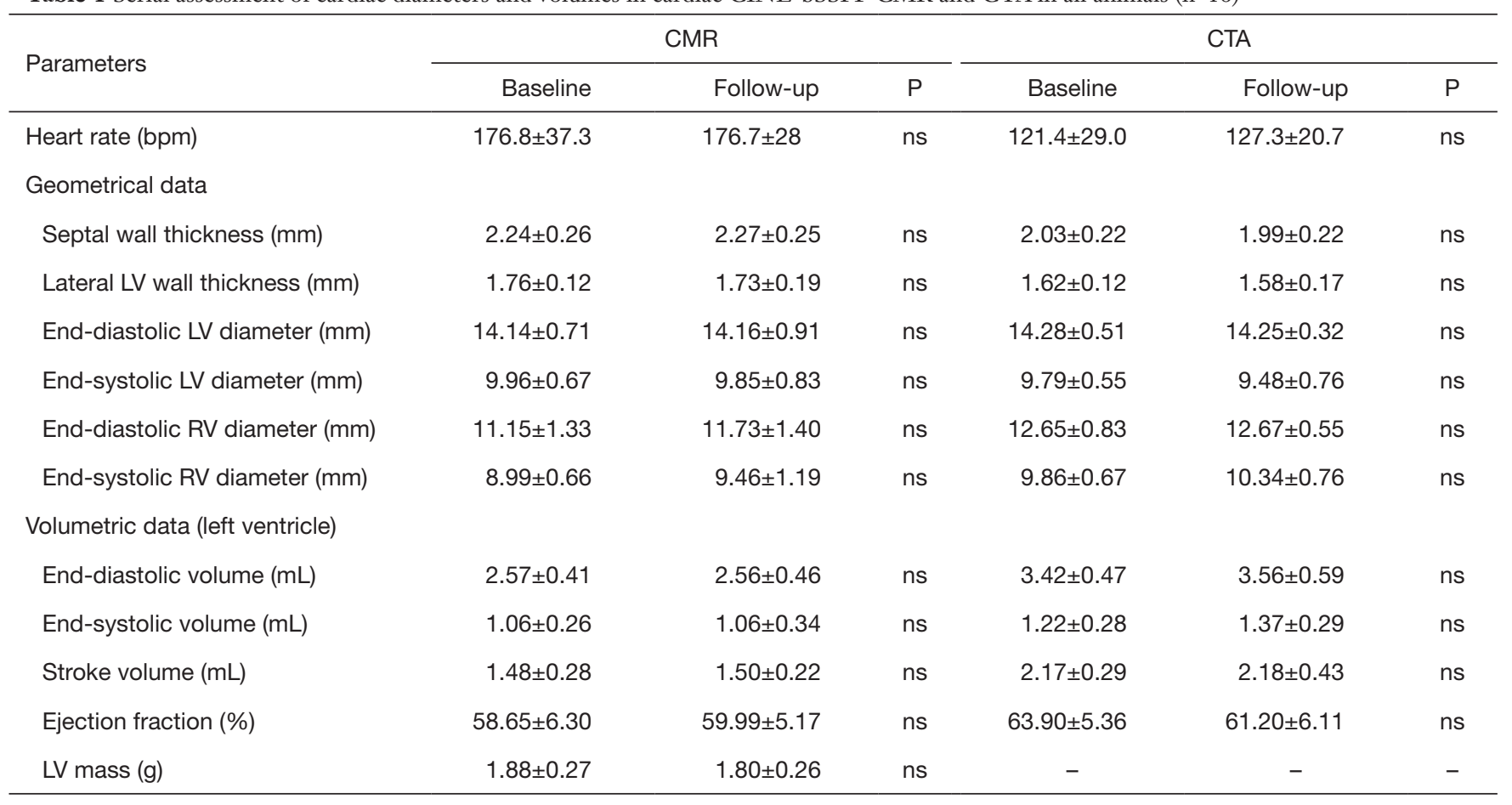

Data are presented as mean \pm standard error of mean (SEM). CMR, cardiac magnetic resonance; CTA, computer tomography angiography; bpm, beats per minute; LV, left ventricle; RV, right ventricle; CTA, cardiac-gated computed tomography angiography; ns, not significant.

order. Details are provided in the Supplementary file.

\section{Statistical analysis}

Statistical analysis was performed using commercially available software (MedCalc ${ }^{\circledR}$ Software, Version 11.4.2.0, Mariakerke, Belgium). Continuous variables are presented as mean or as percentages \pm standard error of mean (SEM), as appropriate. Differences between any two groups (volumes, diameters, thickness) were compared by Student's $t$-test or the Mann-Whitney U test, as appropriate. Continuous variables between more than two groups were compared by one-way ANOVA with post hoc analysis with Bonferroni adjustment for multiple comparisons. All tests were 2-tailed. Correlations were analyzed with linear regression analysis. The differences between the CMR- and CTA-derived measurements and the relationship to the mean were compared with Bland Altman plots, with limits of agreement quoted as $\pm 1.96 \mathrm{SD}$. Intraclass correlation coefficient analysis was performed to assess inter-session agreement of CMR- and CTA-based evaluation of wall thickness, ventricular diameters and volumes. For assessment of inter- and intra-observer variability two independent observers analyzed 10 randomly selected cases and deviations from the initial measurement were calculated (\%). Readings were separated by 8 weeks to minimize recall bias. Differences were considered statistically significant at $\mathrm{P}<0.05$.

\section{Results}

\section{Safety and radiation dose}

Clinical examination and laboratory blood analysis of all animals $(n=16)$ before and 4 days after the application of contrast material excluded any pathologic phenotypes or alterations in blood count, renal and liver function parameters, respectively (Table $S 1$ ). Using retrospective cardiac-gated CTA, total radiation dose (CTDIvol) was $25.3 \pm 0.6 \mathrm{mGy}$.

\section{Image quality}

Cardiac-gated CTA was discontinued in five cases due to heart rates of $>180 / \mathrm{min}$ even after the intravenous application of beta-blockers. Non-diagnostic image quality was observed in 6 of 27 (22\%) CTA versus 8 of 44 (18\%) 
CMR examinations, whereas excellent or good image quality appeared in $78 \%$ of all CTA (21 of 27 ) and in $82 \%$ of all CINE-bSSFP images (36 of 44), respectively ( $\mathrm{P}=0.68$ ). Accurate contrast material bolus tracking and subsequent timing of the acquisition on the one hand, and ECGtriggering and arrhythmia on the other hand were the most important variables causing non-diagnostic image quality with CTA and CMR, respectively.

\section{Assessment of myocardial wall thickness and cardiac diameters}

Values for myocardial wall thickness, $\mathrm{LV}$ and RV diameters can be appreciated in Table S1. Overall, linear regression analysis demonstrated a very good correlation for CMRand CTA-based diameters $(\mathrm{r}=0.9, \mathrm{P}<0.0001$, Figure S1A). Bland-Altman analysis identified systematically higher ventricular diameters in CTA compared to CMR [arithmetic mean of difference (CTA-CMR) $0.66 \mathrm{~mm}, 95 \% \mathrm{CI}$ : 0.40 to 0.91 , limits of agreement -0.84 to $2.15 \mathrm{~mm}$, $\mathrm{P}=0.0005$, Figure $S 1 B]$. On the other hand, CMR revealed systematically higher wall thickness values compared to CTA [arithmetic mean of difference (CTA-CMR) $-0.16 \mathrm{~mm}$, $95 \%$ CI: -0.25 to -0.07 , limits of agreement -0.68 to $0.36 \mathrm{~mm}, \mathrm{P}=0.001]$.

\section{Assessment of LV function, mass and deformation}

All results from volumetric analysis based on (I) CINE-bSSFP, (II) cardiac-gated CTA and (III) velocity-encoded PC-MRI are provided in Table 1. Linear regression analysis showed a good correlation between CTA- and CMR-based volumetric approaches $(\mathrm{r}=0.8, \mathrm{P}<0.0001$, Figure S1C). Bland-Altman analysis demonstrated systematically higher left ventricular (LV) volumes using CTA compared to CMR [arithmetic mean of difference (CTA-CMR) $0.40 \mathrm{~mL}, 95 \% \mathrm{CI}: 0.20$ to 0.66 , limits of agreement -1.5 to $2.4 \mathrm{~mL}, \mathrm{P}<0.001$, Figure S1D]. Wall mass analysis by segmentation of the left ventricle as the volume between the endocardium and epicardium in CINEbSSFP images revealed a mean $L V$ mass of $1.86 \pm 0.26 \mathrm{~g}$.

Using conventional short-axis CINE-bSSFP images we demonstrated the feasibility of commercially available feature tracking software to assess strain and strain rate of the LV in rabbits (Figure S2).

\section{Aortic hemodynamics with $2 D$ velocity-encoded PC-MRI}

$2 \mathrm{D}$ velocity-encoded PC-MRI revealed the following hemodynamic parameters in the ascending aorta: mean velocity: $11.46 \pm 0.41 \mathrm{~cm} / \mathrm{sec}$, stroke volume: $1.50 \pm 0.05 \mathrm{~mL}$, forward flow $1.53 \pm 0.05 \mathrm{~mL}$, backward flow $0.03 \pm 0.01 \mathrm{~mL}$, regurgitation fraction: $1.79 \% \pm 0.25 \%$. Volumetry-based stroke volumes by CINE-bSSFP and CTA revealed only moderate correlation with CINE PC-MRI-based approach (CINE PC-MRI \& CINE-bSSFP: $r=0.48,95 \%$ CI: 0.1 to $0.7, \mathrm{P}=0.01$ ), respectively.

\section{Observer variabilities and inter-session agreement}

Intra- (inter-) observer variabilities for CTA-based wallthickness, ventricular diameter and volume were $96.0 \%$ (99.0\%), 98.2\% (97.4\%) and 98.7\% (98.7\%), respectively. For CMR-based wall-thickness, ventricular diameter and volume intra- (inter-) observer variabilities were $99.1 \%$ (95.6\%), 98.5\% (98.7\%) and 99.9\% (98.8\%), respectively. Correlation analysis revealed a very good correlation (CMR: $\mathrm{r}=0.89, \mathrm{P}<0.001$; CTA: $\mathrm{r}=0.92, \mathrm{P}<0.001)$ and very good intraclass correlation coefficient (CMR: ICC $0.94,95 \%$ CI: 0.92-0.96; CTA: ICC 0.96, 95\% CI: 0.92-0.98) of the diameters measured serially by CMR and CTA at baseline versus follow-up. Serial volume analysis by CMR and CTA also demonstrated a very good correlation (CMR: $\mathrm{r}=0.87$, $\mathrm{P}<0.001$; CTA: $\mathrm{r}=0.96, \mathrm{P}<0.001)$ and very good intraclass correlation coefficient (CMR: ICC 0.93, 95\% CI: 0.82-0.91; CTA: ICC 0.98, 95\% CI: 0.95-0.99) of baseline and followup data (Figure 3, Table 1).

\section{Discussion}

This study demonstrates the ability of clinical-grade CT and MR scanners to provide robust multimodality cardiacgated imaging for the assessment of cardiac morphology, function and aortic hemodynamics in rabbits with a very high inter-session and inter-method agreement.

To apply clinical-grade scanner for preclinical cardiac imaging in rabbits there are some challenges which have to be considered in respect to the specific anatomical and physiological conditions:

(I) What is the appropriate scanner modality (CT vs. $M R)$ in view of the imaging target?

(II) Which technical limitations have to be considered with clinically-equipped CT- or MR-scanners (for example phase array coils or ECG-gating)?

(III) How can particular technical limitations of clinicalgrade scanners be overcome (for example heart rate control) and how may this impact on results? 


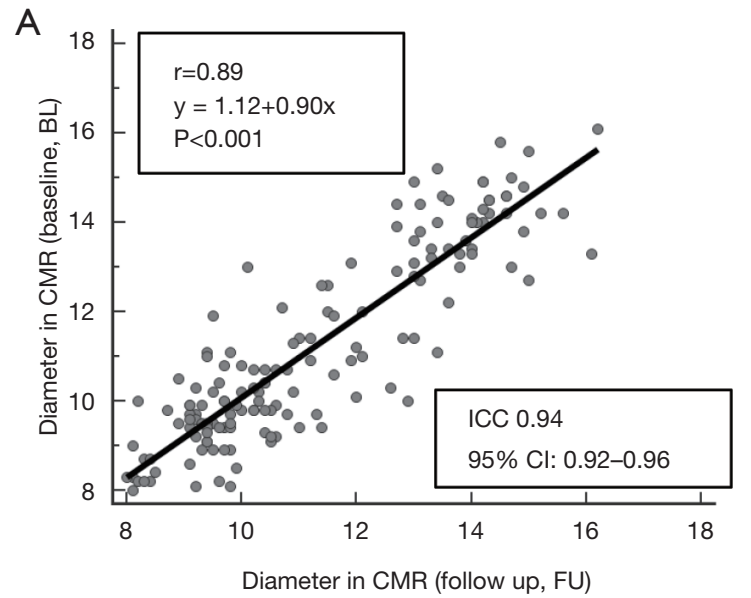

C

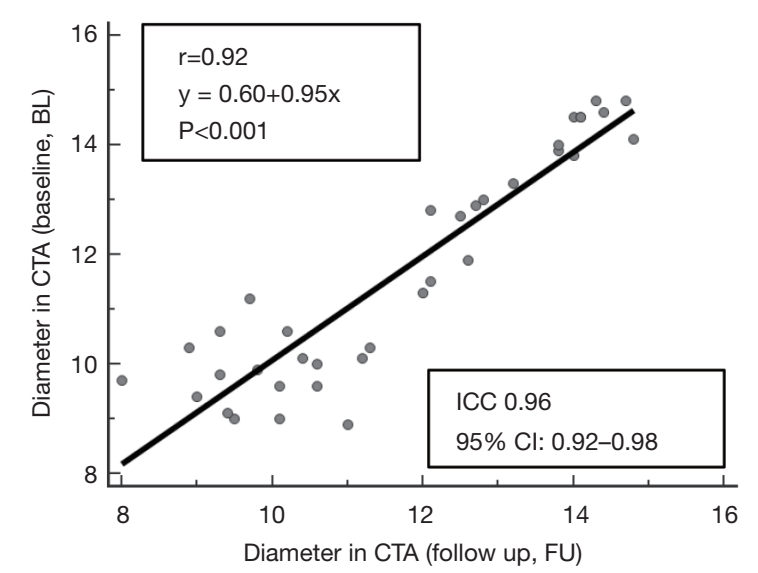

B

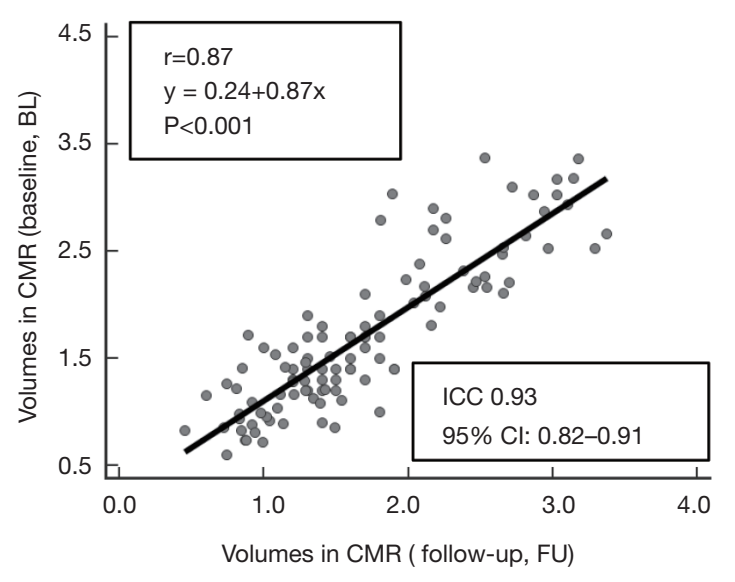

D

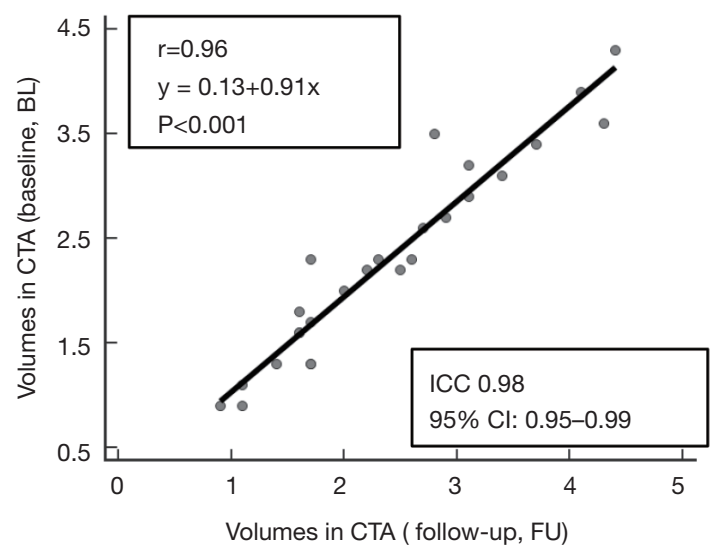

Figure 3 Linear regression analysis of serial CMR (A,B) and CTA (C,D) reveal a very good inter-session agreement for both modalities. ICC, inter-class correlation coefficient; 95\% CI, 95\% confidence interval; CMR, cardiac magnetic resonance; CTA, cardiac-gated computed tomography angiography.

\section{CMR}

The unique feature of CMR is its intrinsic sensitivity enabling for excellent delineation between blood pool and myocardium and quantification of blood flow without the need of ionizing radiation and application of intravascular contrast material. However, performing CMR in rodents requires up to 10 -fold higher spatial resolution than in man. Recent advantages in gradient performance, well-crafted detector geometries and receive array and transmission coils significantly improved sensitivity and acquisition speed, which constituted the major drawbacks with prior scanner generations for application in rodents. Prior small animal studies were mainly performed on MR imagers with ultra- high field strengths ( $\geq 4.7$ Tesla) using dedicated and cost intensive gradient and coil systems $(\$ 10,000$ to $\$ 1,000,000)$ (15-20). However, considering some key items using clinical-grade scanners can significantly increase SNR. First, animal preparation for ECG gating with good quality is necessary, and both body temperature and anesthesia should be monitored and preserved. Signal averaging and novel MR sequences (for example compressed sensing) are effective for increasing SNR and minimizing motion artifacts, while reducing acquisition time. Finally, higher field strength innately provides increased SNR (15). Our results confirm the ability of clinically-equipped $M R$ scanners for robust cardiac assessment in rabbits (22-24) and additionally demonstrate a strong robustness for serial 
imaging. Difficult ECG triggering (arrhythmia or reduced ECG quality) represents the major drawback which impacts on reduced image quality.

\section{CINE PC-MRI}

Uniquely, velocity encoding CINE PC-MRI provides the most accurate method available to assess cardiac output and measurements of aortic, pulmonary and mitral valve regurgitations, all non-invasively and without ionizing radiation and contrast material (25-28). Cardiac-gated time-resolved 3D CINE PC-MRI allows for registration of blood flow in cardiac chambers and in targeted arteries $(29,30)$, however, with limited applicability due to very time-consuming acquisition protocols (31). In the present study, we demonstrate that a standard $2 \mathrm{D}$ velocity encoding CINE PC-MRI protocol enables for robust assessment of aortic blood flow and ventricular stroke volume in rabbits. The moderate correlation to CINE-bSSFP-based volumes could be caused by encoding only the throughplane velocity vector component and high slice-thickness to achieve sufficient SNR.

\section{Cardiac-gated CTA}

Small-animal cardiac-gated CTA yet plays a subordinate role for the assessment of cardiac morphology and function in rodents, which can be accounted for limited temporal and spatial resolutions with prior CT scanner generations. Therefore, limited data exist on the use of clinical-grade CT scanners for application in rodents. Our results demonstrate the ability of a clinical-grade 256-slice CT scanner to provide a robust tool with excellent intersession agreement for cardiac imaging in rabbits. Above, this study directly compares the serial evaluation of cardiac morphology and function as assessed by clinical-grade CT versus MR scanners in rabbits. Even though, cardiac-gated CTA revealed systematically higher ventricular diameters and volumes compared to CMR (12), correlation analysis revealed a very good association of diameters and volumes as evaluated by CTA and CMR. Acquisition time plays an important role for a high-volume routine application in preclinical imaging studies. Cardiac-gated CTA requires 4-5 times less acquisition time as compared to CINEbSSFP and provides highly reproducible measurement of LV volumes (12). Thus, the presented protocols could facilitate the applicability of cardiac-gated CT imaging in preclinical rabbit studies focusing on cardiac volumes and function.

The radiation dose applied to the animals during CT imaging is often seen as a limitation, especially for serial examinations. Previously, cardiac-gated CTA studies mainly investigated on pre-clinical flat-panel or micro-CT scanners reported a dose of $~ 90 \mathrm{mGy}$ (32-36). Using a clinical-grade 256-slice CT scanner with retrospective ECG-gating, we achieved a mean dose of $25 \mathrm{mGy}$, which cannot be expected to significantly affect the health of animals, even if applied serially. A critical limitation with CTA is the need for beta-blockers to maintain low heart rates, which may impact on the evaluation of $\mathrm{LV}$ function due to its negative inotropic effects.

\section{Deformation analysis}

Off-line feature tracking for cardiac deformation analysis is established to provide deeper understanding into the pathophysiology of myocardial ischemia and infarction, cardiomyopathies and the impact of valvular diseases on myocardial function (37). Our results are promising that feature tracking could be feasible in cardiac imaging studies using clinical-grade CT and MR.

\section{Limitations}

Our study has some limitations. All animals in our study were healthy, which implicates the necessity of further investigations in animal models with ischemic or primary myocardial dysfunction or valvular or vessel disease. Under anesthesia we noticed only small intrathoracic excursion during breathing. However, CMR and CTA imaging was performed without any respiratory gating. To overcome this limitation, we implemented signal averaging with overall good image quality. Beta-blocker application prior to CTA scans may impact on the evaluation of LV function because of its negative inotropic effect. Finally, our study included only rabbits. Further investigations are needed to demonstrate the applicability in rodents.

\section{Conclusions}

In conclusion, multimodality non-invasive assessment of cardiac morphology, function and aortic hemodynamics is feasible and robust in rabbits using clinical-grade MR and CT imagers and provide results with an excellent intersession agreement for. The presented imaging protocols for preclinical studies could facilitate the applicability of 
MR- and CT-based cardiac assessment, especially for serial disease monitoring under therapeutically interventions.

\section{Acknowledgments}

None.

\section{Footnote}

Conflicts of Interest: The authors have no conflicts of interest to declare.

Ethical Statement: The authors are accountable for all aspects of the work in ensuring that questions related to the accuracy or integrity of any part of the work are appropriately investigated and resolved. The study was approved by the institutional Animal Care and Use Committee (approval number: 35-9185.81/G-39/09).

\section{References}

1. Brown WH, Pearce L, Van Allen CM. Organ weights of normal rabbits. J Exp Med 1925;42:69-82.

2. Litwin SE, Katz SE, Morgan JP, et al. Serial echocardiographic assessment of left ventricular geometry and function after large myocardial infarction in the rat. Circulation 1994;89:345-54.

3. Collins KA, Korcarz CE, Shroff SG, et al. Accuracy of echocardiographic estimates of left ventricular mass in mice. Am J Physiol Heart Circ Physiol 2001;280:H1954-62.

4. Weytjens C, Cosyns B, D'Hooge J, et al. Doppler myocardial imaging in adult male rats: reference values and reproducibility of velocity and deformation parameters. Eur J Echocardiogr 2006;7:411-7.

5. Jugdutt BI, Schwarz-Michorowski BL, Khan MI. Effect of long-term captopril therapy on left ventricular remodeling and function during healing of canine myocardial infarction. J Am Coll Cardiol 1992;19:713-21.

6. Rottman JN, Ni G, Brown M. Echocardiographic evaluation of ventricular function in mice. Echocardiography 2007;24:83-9.

7. Stypmann J, Engelen MA, Troatz C, et al. Echocardiographic assessment of global left ventricular function in mice. Lab Anim 2009;43:127-37.

8. Ghanem A, Roll W, Hashemi T, et al. Echocardiographic assessment of left ventricular mass in neonatal and adult mice: accuracy of different echocardiographic methods.
Echocardiography 2006;23:900-7.

9. Dawson D, Lygate CA, Saunders J, et al. Quantitative 3-dimensional echocardiography for accurate and rapid cardiac phenotype characterization in mice. Circulation 2004;110:1632-7.

10. Paulus MJ, Gleason SS, Easterly ME, et al. A review of high-resolution $\mathrm{X}$-ray computed tomography and other imaging modalities for small animal research. Lab Anim (NY) 2001;30:36-45.

11. Miller GG, Dawson DT, Battista JJ. Computed tomographic assessment of radiation induced damage in the lung of normal and WR 2721 protected LAF1 mice. Int J Radiat Oncol Biol Phys 1986;12:1971-5.

12. Sugeng L, Mor-Avi V, Weinert L, et al. Quantitative assessment of left ventricular size and function: sideby-side comparison of real-time three-dimensional echocardiography and computed tomography with magnetic resonance reference. Circulation 2006;114:654-61.

13. Oppelt A, Graumann R, Barfuss H, et al. FISP - eine neue schnelle Pulssequenz für die Kernspintomographie. Electromedica 1986;54:15-8.

14. Pelc NJ. Flow quantification and analysis methods. Magn Reson Imaging Clin N Am 1995;3:413-24.

15. Gilson WD, Kraitchman DL. Cardiac magnetic resonance imaging in small rodents using clinical $1.5 \mathrm{~T}$ and $3.0 \mathrm{~T}$ scanners. Methods 2007;43:35-45.

16. Epstein FH. MR in mouse models of cardiac disease. NMR Biomed 2007;20:238-55.

17. Chapon C, Herlihy AH, Bhakoo KK. Assessment of myocardial infarction in mice by late gadolinium enhancement $M R$ imaging using an inversion recovery pulse sequence at 9.4T. J Cardiovasc Magn Reson 2008;10:6.

18. Oshinski JN, Yang Z, Jones JR, et al. Imaging time after Gd-DTPA injection is critical in using delayed enhancement to determine infarct size accurately with magnetic resonance imaging. Circulation 2001;104:2838-42.

19. Klug G, Kampf T, Ziener C, et al. Murine atherosclerotic plaque imaging with the USPIO Ferumoxtran-10. Front Biosci (Landmark Ed) 2009;14:2546-52.

20. Berry CJ, Miller JD, McGroary K, et al. Biventricular adaptation to volume overload in mice with aortic regurgitation. J Cardiovasc Magn Reson 2009;11:27.

21. Lu JG, Lv B, Chen XB, et al. What is the best contrast injection protocol for 64-row multi-detector cardiac computed tomography? Eur J Radiol 2010;75:159-65. 
22. Voelkl JG, Haubner BJ, Kremser C, et al. Cardiac imaging using clinical $1.5 \mathrm{t}$ MRI scanners in a murine ischemia/reperfusion model. J Biomed Biotechnol 2011;2011:185683.

23. Franco F, Dubois SK, Peshock RM, et al. Magnetic resonance imaging accurately estimates LV mass in a transgenic mouse model of cardiac hypertrophy. Am J Physiol 1998;274:H679-83.

24. Franco F, Thomas GD, Giroir B, et al. Magnetic resonance imaging and invasive evaluation of development of heart failure in transgenic mice with myocardial expression of tumor necrosis factor-alpha. Circulation 1999;99:448-54.

25. Kilner PJ, Gatehouse PD, Firmin DN. Flow measurement by magnetic resonance: a unique asset worth optimising. J Cardiovasc Magn Reson 2007;9:723-8.

26. Petersen SE, Voigtlander T, Kreitner KF, et al. Quantification of shunt volumes in congenital heart diseases using a breath-hold MR phase contrast technique-comparison with oximetry. Int J Cardiovasc Imaging 2002;18:53-60.

27. Gelfand EV, Hughes S, Hauser TH, et al. Severity of mitral and aortic regurgitation as assessed by cardiovascular magnetic resonance: optimizing correlation with Doppler echocardiography. J Cardiovasc Magn Reson 2006;8:503-7.

28. Nayler GL, Firmin DN, Longmore DB. Blood flow imaging by cine magnetic resonance. J Comput Assist Tomogr 1986;10:715-22.

29. Markl M, Harloff A, Bley TA, et al. Time-resolved 3D

Cite this article as: Gitsioudis G, Nunninger M, Missiou A, Wolf P, Katus HA, Korosoglou G. Multimodality cardiac computed tomography angiography and magnetic resonance with clinical-grade scanners provide robust assessment of cardiac morphology and function in rabbits. $\mathrm{J}$ Thorac Dis 2019;11(11):4762-4771. doi: 10.21037/jtd.2019.10.46
MR velocity mapping at 3T: improved navigator-gated assessment of vascular anatomy and blood flow. J Magn Reson Imaging 2007;25:824-31.

30. Wigstrom L, Sjoqvist L, Wranne B. Temporally resolved 3D phase-contrast imaging. Magn Reson Med 1996;36:800-3.

31. Ebbers T. Flow Imaging: Cardiac Applications of 3D Cine Phase-Contrast MRI. Curr Cardiovasc Imaging Rep 2011;4:7.

32. Dinkel J, Bartling SH, Kuntz J, et al. Intrinsic gating for small-animal computed tomography: a robust ECGless paradigm for deriving cardiac phase information and functional imaging. Circ Cardiovasc Imaging 2008;1:235-43.

33. Badea CT, Fubara B, Hedlund LW, et al. 4-D micro-CT of the mouse heart. Mol Imaging 2005;4:110-6.

34. Ford NL, Nikolov HN, Norley CJ, et al. Prospective respiratory-gated micro-CT of free breathing rodents. Med Phys 2005;32:2888-98.

35. Buliev IG, Badea CT, Kolitsi Z, et al. Estimation of the heart respiratory motion with applications for cone beam computed tomography imaging: a simulation study. IEEE Trans Inf Technol Biomed 2003; 7:404-11.

36. Drangova M, Ford NL, Detombe SA, et al. Fast retrospectively gated quantitative four-dimensional (4D) cardiac micro computed tomography imaging of freebreathing mice. Invest Radiol 2007;42:85-94.

37. Gorcsan J 3rd, Tanaka H. Echocardiographic assessment of myocardial strain. J Am Coll Cardiol 2011;58:1401-13. 


\section{Animal preparation and monitoring during CMR imaging}

Animals received ketamine $(50 \mathrm{mg} / \mathrm{kg})$ and xylazine $(20 \mathrm{mg} / \mathrm{kg}$ ) intramuscularly for general anesthesia during all imaging studies. Blood was taken from the left marginal ear vein from all animals for analysis of renal and hepatic function 4 days after the application of contrast material. Earplugs were placed in each external auditory canal for inner ear protection. Studies were approved by the institutional Animal Care and Use Committee (approval number: 35-9185.81/G-39/09).

\section{CMR imaging protocols}

Essential, to achieve high spatial resolution without relevant image degradation due to cardiac motion, the MR system needs fast switching and strong gradients. We therefore used the gradient equipment of the MR scanner, which achieved a maximum slew rate (on axis) of $180 \mathrm{Tesla} / \mathrm{m} / \mathrm{s}$ and a maximum gradient strength (on axis) of $66 \mathrm{mTes} \mathrm{la} / \mathrm{m}$.

All CINE images were acquired using a balanced steadystate free precession (b-SSFP) sequence in combination with parallel imaging (SENSitivity Encoding, factor 2) and retrospective vector-ECG gating. Figure $2 A$ illustrates a timing diagram for a CINE-bSSFP acquisition. The following imaging parameters were used: Echo time (TE) 2.2-2.4 ms; repetition time (TR) 4.4-5.1 ms; flip angle (FA) $60^{\circ}$ (spatial resolution, $2.4 \mathrm{~mm} \times 2.5 \mathrm{~mm}$; slice thickness: 3-5 mm; 35 phases per cardiac cycle).

2D CINE velocity-encoded phase-contrast MR imaging (CINE PC-MRI) was performed using retrospective vector-ECG gated CINE imaging with through-plane velocity encoding. Time-resolved pulsatile aortic blood flow was measured perpendicular to the aorta at the level of the proximal ascending aorta (Figure S3). The following imaging parameters were used: TR $4.0 \mathrm{~ms}$; TE $2.0 \mathrm{~ms}$; FA $15^{\circ}$; slice thickness $8-10 \mathrm{~mm}$; spatial resolution $1.5 \mathrm{~mm}$ $\times 2.5 \mathrm{~mm}$. To avoid velocity aliasing and for optimal signal intensity, velocity encoding sensitivity (VENC) was adjusted at $50-200 \mathrm{~cm} / \mathrm{sec}(38)$, as appropriate.

\section{CMR image analysis}

Left and right ventricular end-diastolic and end-systolic diameters $[\mathrm{LV} / \mathrm{RV}-\mathrm{E}(\mathrm{D} / \mathrm{S}) \mathrm{D}]$ were assessed in 4-chamber view. Septal (SeptalWT) and lateral (LatWT) LV wall thickness was assessed in short-axis views (39). Endocardial
LV borders were manually traced at end-diastole and endsystole, and the papillary muscles were excluded (Figure S4). $\mathrm{LV}$ End-diastolic and endsystolic volumes $[\mathrm{LV}-\mathrm{E}(\mathrm{D} / \mathrm{S}) \mathrm{V}]$ were determined and $L V$ ejection fraction (LVEF) was computed as:

$L V E F(\%)=[(L V E D V-L V E S V) / L V E D V] \times 100 \%$

For LV deformation analysis (strain and strain rate) CINE-bSSFP short-axis views were analyzed. Retrospective image analyses were conducted using the 2D feature tracking software (TomTec Imaging Systems, Munich, Germany).

Blood flow quantification in $2 \mathrm{D}$ velocity-encoded PC-MRI was performed using commercially available cardiovascular software (View Forum, Version 5.1, Philips Medical Systems, Best, the Netherlands). Regions of interest were drawn in the proximal ascending aorta. All measurements were performed by 2 independent and experienced investigators (M Nunninger, G Gitsioudis).

\section{Animal preparation and monitoring for CT imaging}

Animal preparation included the intravenous insertion of a saline-filled angiocath into the left marginal ear vein for drug administration and contrast-enhanced illumination of the heart and the thoracic aorta. Animals with heart rates $\geq 180$ beats/min received an intravenous administration of incremental doses of metoprolol (range, 2.5-10.0 mg, Lopresor ${ }^{\circledR}$, Novartis, Pharma GmbH, Germany) 10-15 min before the CT scan to achieve the lowest possible and regular heart rate to optimize ECG for cardiac-gated CTA acquisition.

\section{CT image analysis}

Quantitative analysis included LV-E(D/S)D, SeptalWT, InfWT and threshold based LV segmentation [LV-E(D/ $\mathrm{S}) \mathrm{V}]$ for the assessment of the LVEF according to the formula Eq. [1]. All measurements were performed by 2 independent and experienced investigators (M Nunninger, G Gitsioudis).

\section{Assessment of image quality}

CMR and CTA data sets were analyzed in random order. Image quality was assessed in both end-systolic and enddiastolic images, with adjusted window setting. Image quality was determined by consensus of two experienced 
observers (M Nunninger, G Gitsioudis) on the basis of the presence of motion artifacts using a 5 -grade scale: ' 1 ': Excellent image quality, i.e., no motion artifacts; '2': good image quality, i.e., minimal motion artifacts; ' 3 ': moderate image quality, i.e., mild motion artifacts; '4': poor image quality, i.e., moderate motion artifacts; ' 5 ': extremely poor, non-diagnostic quality, due to severe motion artifacts.

\section{References}

38. Menon A, Wendell DC, Wang H, et al. A coupled experimental and computational approach to quantify deleterious hemodynamics, vascular alterations, and mechanisms of long-term morbidity in response to aortic coarctation. J Pharmacol Toxicol Methods 2012;65:18-28.

39. Puntmann VO, Gebker R, Duckett $S$, et al. Left ventricular chamber dimensions and wall thickness by cardiovascular magnetic resonance: comparison with transthoracic echocardiography. Eur Heart J Cardiovasc Imaging 2013;14:240-6. 
Table S1 Overview of all $(\mathrm{n}=16)$ basic animal characteristics and laboratory data

\begin{tabular}{|c|c|c|c|}
\hline Parameters & Before contrast material application & After contrast material application & $\mathrm{P}$ \\
\hline Serum urea $(\mathrm{mg} / \mathrm{dL})$ & $39.15 \pm 1.34$ & $38.47 \pm 1.69$ & 0.74 \\
\hline Hs-TnT (pg/mL) & $12.76 \pm 1.62$ & $10.00 \pm 1.25$ & 0.19 \\
\hline Leukocytes (/nL) & $3.93 \pm 0.24$ & $4.69 \pm 0.35$ & 0.08 \\
\hline AST (U/L) & $29.41 \pm 2.74$ & $32.15 \pm 2.47$ & 0.46 \\
\hline $\operatorname{ALT}(\mathrm{U} / \mathrm{L})$ & $71.44 \pm 6.00$ & $89.30 \pm 6.38$ & 0.08 \\
\hline
\end{tabular}

Data are presented as mean \pm standard error of mean (SEM). Hs-TnT, high-sensitive troponin T; AST, aspartate aminotransferase; ALT, alanine aminotransferase.

A

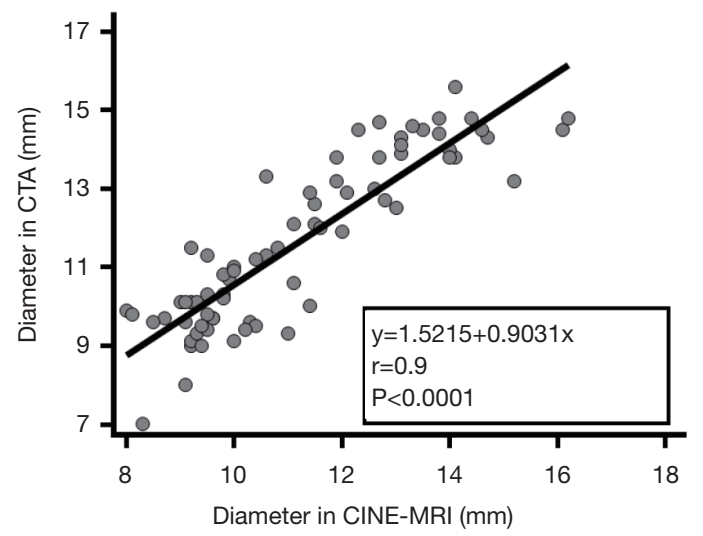

C

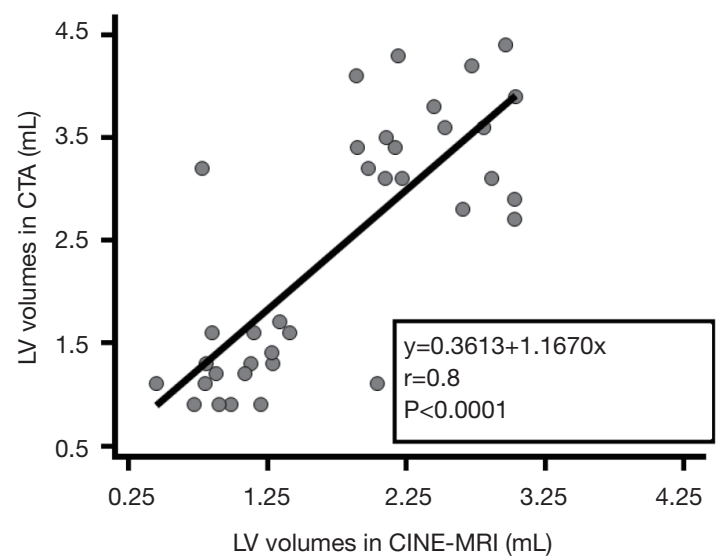

B

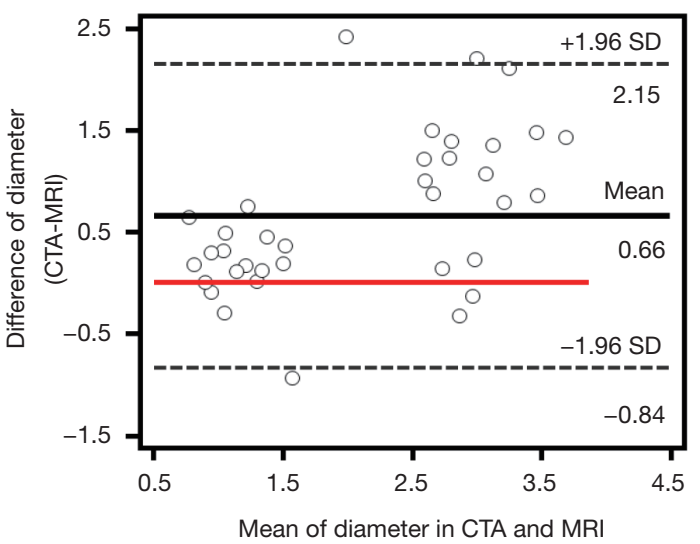

D

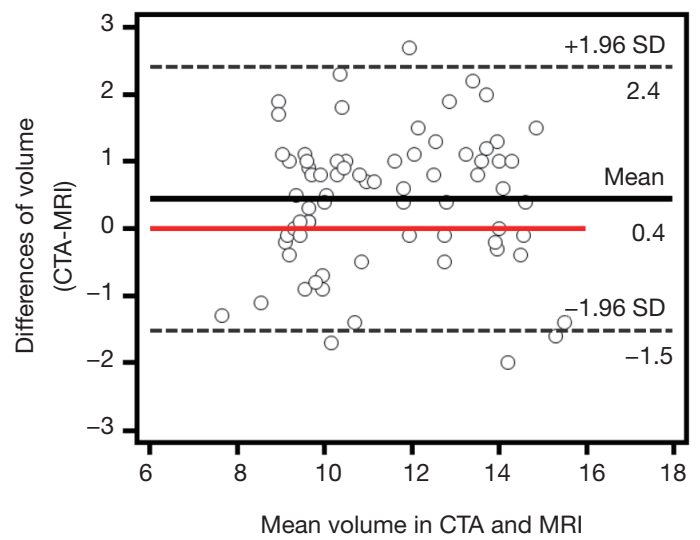

Figure S1 Linear regression analysis demonstrates a very good correlation for CMR- and CTA-based diameters and volumes (A,C). BlandAltman analysis reveals a systematic overestimation for the assessment of ventricular diameters and volumes in CTA compared to CMR (B,D). CMR, cardiac magnetic resonance; CTA, cardiac-gated computed tomography angiography. 


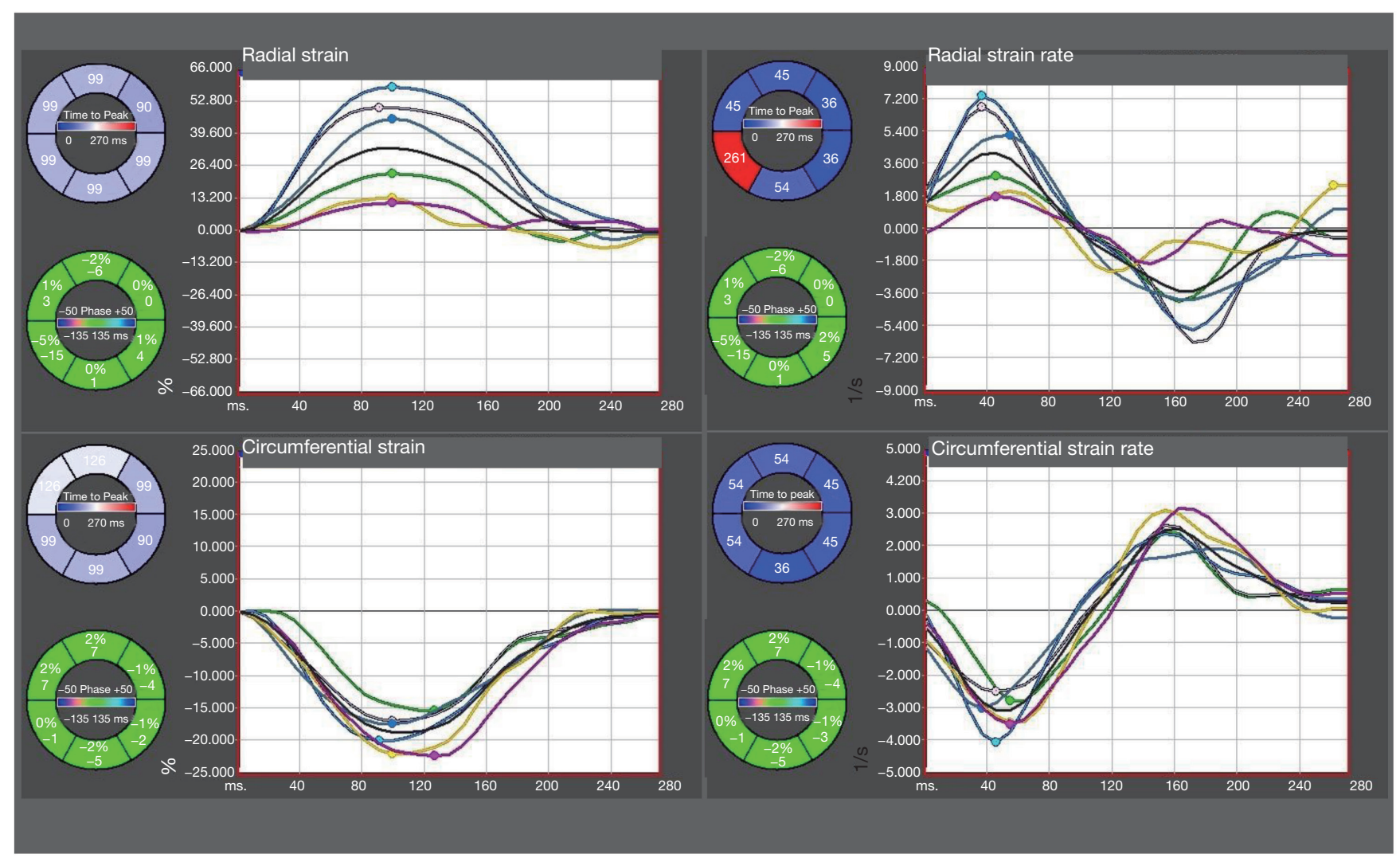

Figure S2 Feature tracking using CINE-bSSFP CMR imaging showing the curves of radial strain and strain rate. CMR, cardiac magnetic resonance.
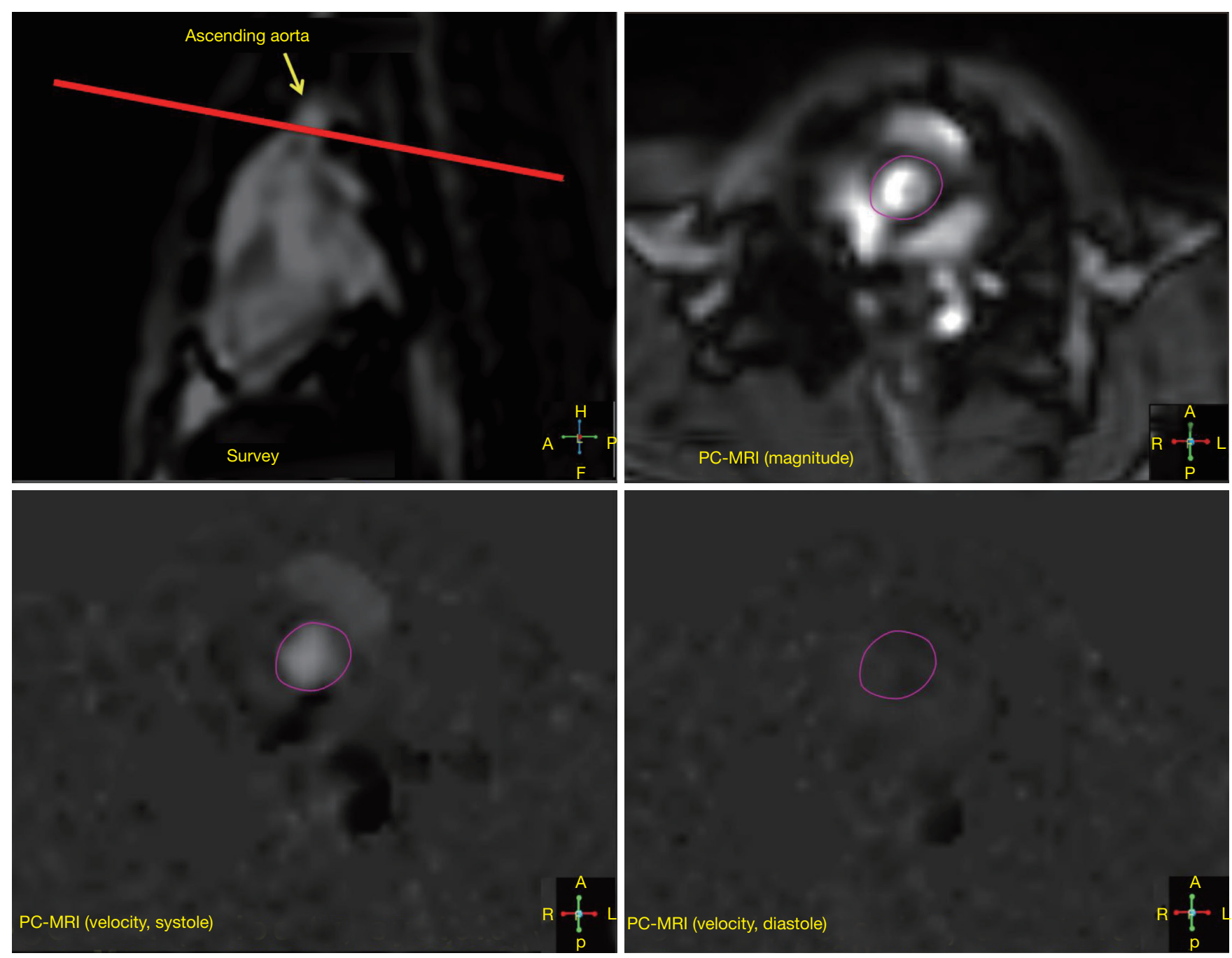

Figure S3 Aortic blood flow was measured perpendicular to the aorta at the level of aortic bulb proximal ascending aorta (survey). A representative magnitude image and the corresponding phase images from $2 \mathrm{D}$ velocity encoding PC-MRI are provided. PC-MRI, phasecontrast magnetic resonance imaging. 

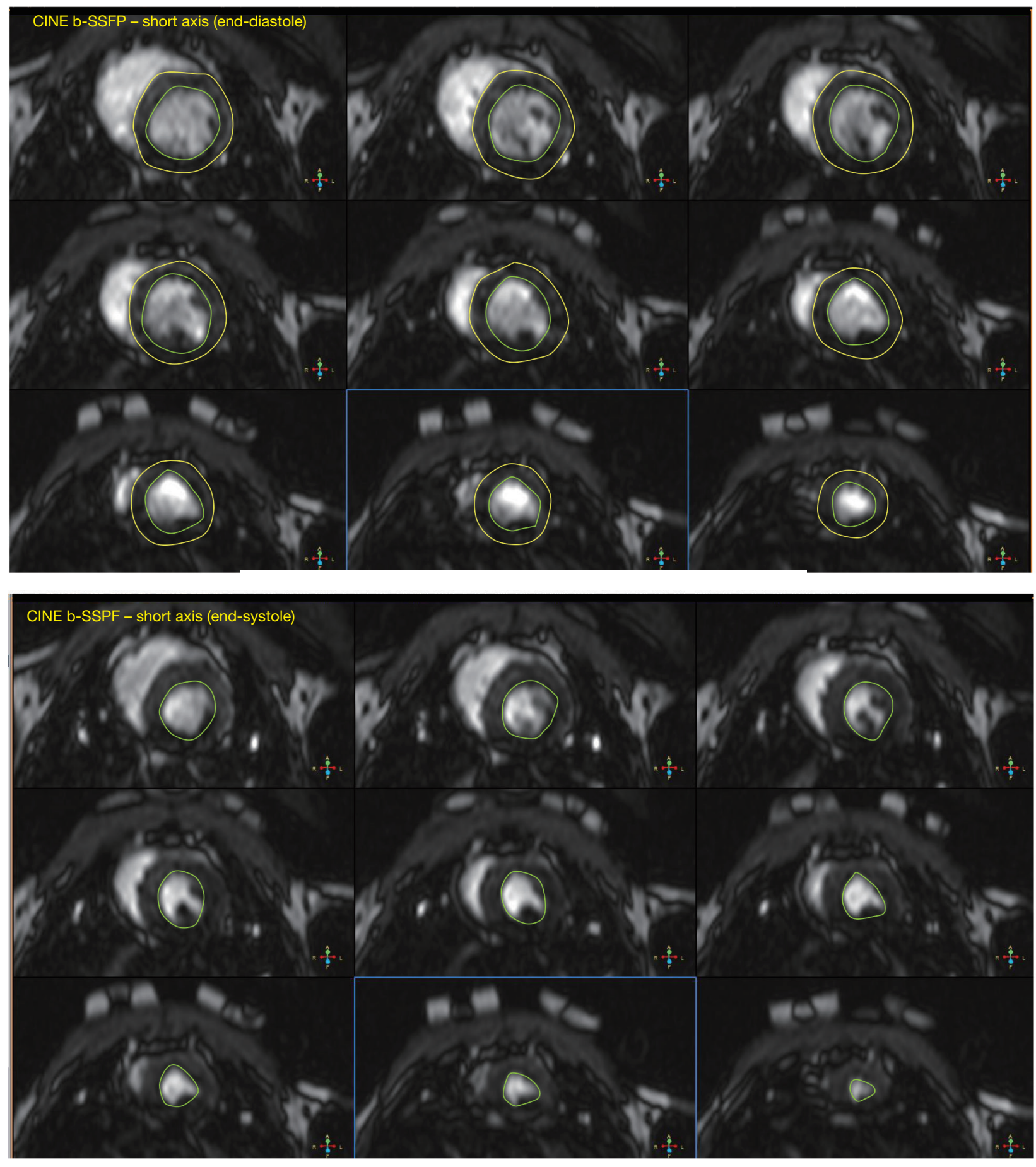

Figure S4 Manual traced segmentation of the endocardial LV borders at end-diastole (above) and end-systole (below), with exclusion of the papillary muscles. LV, left ventricular. 